\title{
Digitale Prozessüberwachung in der Lackiererei
}

\author{
Neue Software für den perfekten Lack: Experten verschiedener Fachbereiche am Audi-Standort \\ Neckarsulm haben gemeinsam das sogenannte „Paintshop Cockpit" entwickelt. Damit lassen sich \\ Abweichungen im Lackiervorgang frühzeitig erkennen und durch Anpassungen im Prozess \\ beseitigen.
}

Der Audi-Standort Neckarsulm bietet Kunden für ihr neues Fahrzeug eine große Auswahl an Farbvariationen. Neben den Standardfarben der Marke werden auch Individuallackierungen speziell auf Kundenwunsch angefertigt. Zusammen mit der großen Modellvielfalt am Standort wird das Lackieren zu einem sehr komplexen Verfahren und erfordert eine besonders gute Prozessüberwachung.

Bei diesem wichtigen Schritt werden die Mitarbeiter durch eine neue Software unterstützt: Das Paintshop Cock- pit (PSC) bündelt, sortiert und prüft Daten aus dem Lackiervorgang und hilft bei der Prozessüberwachung, mögliche Abweichungen frühzeitig zu erkennen. Dafür werden Auffälligkeiten wie kleinste Schmutzpartikel oder Luftbläschen im Lack direkt an der Linie in ein bereits bestehendes IT-System, die sogenannte elektronische Wagenbegleitkarte, eingetragen. Die dort gespeicherten Daten werden dann zusammen mit der Schichtzeit, der Lackfarbe und dem Fahrzeugmodell im PSC ausgewertet.



Die Mitarbeiter in der Lackiererei können Abweichungen im Lack direkt an der Linie in ein Erfassungssystem eingeben. Alle Daten werden anschließend gebündelt und ausgewertet.

\section{Genau Datenanalyse ermöglicht gezielte Anpassungen}

Alle Informationen werden permanent vom System aktualisiert und regelmäßig von Experten kontrolliert, was eine genaue Datenanalyse ermöglicht. In einem nächsten Schritt können die Mitarbeiter dann gezielt Anpassungen im Lackierprozess vornehmen, wie beispielsweise die Lackierroboter umprogrammieren.

Die Software wird stetig weiterentwickelt und soll zukünftig sowohl in der Lackiererei in Ingolstadt als auch in anderen Fachbereichen zum Einsatz kommen. Entstanden ist das Paintshop Cockpit in Zusammenarbeit verschiedener Fachbereiche der Audi AG. Beteiligt waren neben der Prozesssicherung die Bereiche Instandhaltung, IT und das Team TechHub Data Driven Production Heilbronn, das sich seit 2017 gezielt um Projekte im Bereich Data Analytics in der Produktion kümmert. //

\section{Kontakt}

Audi AG Werk Neckarsulm

Neckarsulm

www.audi.de 